\title{
Stability properties and probability distribution of multi-overlaps in dilute spin glasses
}

\author{
Adriano Barra *, Luca De Sanctis ${ }^{\dagger}$
}

March 9, 2019

\begin{abstract}
We prove that the Aizenman-Contucci relations, well known for fully connected spin glasses, hold in diluted spin glasses as well. We also prove more general constraints of the same spirit for multioverlaps, confirming previous results. The strategy we employ makes no use of self-averaging, and allows to generate systematically all these relations within the framework of Random Multi-Overlap Structures. The basic idea is to study, for these structures, the consequences of the strictly related concepts of stochastic stability, quasistationarity under random shifts, factorization properties of the trial free energy. The very simple technique allows to prove also the phase transition for multi-overlaps: they remain strictly positive (in average) below their critical temperature if a suitable external field is first applied and then removed in the thermodynamic limit. We also deduce, from a cavity approach, the general form of the constraints
\end{abstract}

\footnotetext{
*King's College London, Department of Mathematics, Strand, London WC2R 2LS, United Kingdom, and Dipartimento di Fisica, Università di Roma "La Sapienza" Piazzale Aldo Moro 2, 00185 Roma, Italy, <Adriano.Barra@roma1.infn.it>

${ }^{\dagger}$ ICTP, Strada Costiera 11, 34014 Trieste, Italy, <lde_sanc@ictp.it>
} 
on the distribution of multi-overlaps found within Quasi-Stationary

Random Multi-Overlap Structures, and give a simple proof of the self-averaging that leads to the Aizenman-Contucci relations, showing that it is in turn deducible from stochastic stability.

Key words and phrases: spin glasses, diluted spin glasses, Aizenman-Contucci relations, Quasi-Stationary Random Multi-Overlap Structures.

\section{Introduction}

Dilute spin glasses are studied mostly for two reasons: their finite connectivity makes them in some sense close to finite-dimensional systems, though being still mean field; they are mathematically equivalent to some important random optimization problems (such as the X-OR-SAT and K-SAT [15]). The proper setting for the study of mean field dilute spin glasses are the Random Multi-Overlap Structures (RaMOSt), and the whole physical content of dilute spin glasses is carried by the probability distribution of the multi-overlaps [7, like the 2-overlap does for fully connected models. As per the latter, it is known that the Ghirlanda-Guerra identities [12] allow for the computation of the critical exponents governing the critical behavior of the 2-overlap [1], and guarantee that the 2-overlap is positive below the critical temperature [15]. The relations due to Aizenman and Contucci [2], instead, imply [1] that the expectation of the 2-overlap is strictly positive below the critical temperature (due to a phase transition triggered by an external field). Ghirlanda-Guerra identities are a consequence of the self-averaging of the energy density, and extend to dilute spin glasses, where one can find more general relations for multi-overlaps too [9]. Aizenman-Contucci (AC) relations are instead a consequence of stochastic stability [2, 6, but they are also implied by a certain self-averaging, as shown by Franz et al. [10, who extended the AC relations to dilute spin glasses and multi-overlaps. In this paper we provide a new proof of $\mathrm{AC}$ relations for dilute spin glasses, and of the relations for multi-overlaps. We show that stochastic stability 
(and connected properties like quasi-stationarity of Random Multi-Overlap Structures) plays a key role in dilute spin glasses too, as they imply the AC relations and generalizations to multi-overlaps. We emphasize that the concepts of stochastic stability (and similar concepts) is intimately related to self-averaging properties. Moreover, both approaches can be used with observables of various forms, not just the with the energy. The joint use of the the techniques developed in [1] within the approach developed in [7] is at the basis of the present work. The latter is thus organized as follows. The next two sections introduce the model and the notations, and illustrate the cavity perspective which the RaMOSt approach relies on. In section 4 we show that simple symmetry arguments within the cavity method lead to the proof of the phase transition of the the expectation of the multioverlaps: below its critical temperature each multi-overlap remains strictly positive, if an external (cavity) field is applied and then removed in the thermodynamic limit. Section 5 is devoted to a proof that AC relations hold in dilute glasses, where some further relations of the same spirit are found for multi-overlaps. We already stressed that our proof is radically different from the one hinted at in [10], and systematically developed in 9. Section 6 presents the form of the derivative with respect to a perturbing parameter of the expectation of a generic function of some replicas. The result makes it possible to develop systematically the constraints on multi-overlaps, whose critical behavior control can be here improved as compared to section 4 (although the critical exponents are not found yet). In Section 6, we also sketch a comparison between stochastic stability and self-averaging, the latter being proven in a simple way in the case considered in [10]. The straightforward but tedious and long calculations needed in some expansions are reported in the appendices, preceded by concluding remarks. 


\section{Model and notations}

Consider $N$ points, indexed by latin letters $i, j$, etc., with an Ising spin attached to each of them, so to have spin configurations

$$
\sigma:\{1, \ldots, N\} \ni i \rightarrow \sigma_{i}= \pm 1
$$

Hence $\sigma \in\{-1,+1\}^{N}$. Let $P_{\zeta}$ be a Poisson random variable of mean $\zeta$, let $\left\{J_{\nu}\right\}$ be independent identically distributed copies of a random variable $J$ with symmetric distribution. For the sake of simplicity we will assume $J= \pm 1$, without loss of generality [8]. We want to consider randomly chosen points, we therefore introduce $\left\{i_{\nu}\right\},\left\{j_{\nu}\right\}$ as independent identically distributed random variables, with uniform distribution over $1, \ldots, N$. Assuming there is no external field, the Hamiltonian of the Viana-Bray (VB) model of dilute mean field spin glass is the following symmetric random variable

$$
H_{N}(\sigma, \alpha ; \mathcal{J})=-\sum_{\nu=1}^{P_{\alpha N}} J_{\nu} \sigma_{i_{\nu}} \sigma_{j_{\nu}}, \alpha \in \mathbb{R}_{+} .
$$

$\mathbb{E}$ will be the expectation with respect to all the (quenched) variables, i.e. all the random variables but the spins, collectively denoted by $\mathcal{J}$. The nonnegative parameter $\alpha$ is called degree of connectivity. The Gibbs measure $\omega$ is defined by

$$
\omega(\varphi)=\frac{1}{Z} \sum_{\sigma} \exp (-\beta H(\sigma)) \varphi(\sigma)
$$

for any observable $\varphi:\{-1,+1\}^{N} \rightarrow \mathbb{R}$, and clearly

$$
Z_{N}(\beta)=\sum_{\sigma} \exp \left(-\beta H_{N}(\sigma)\right)
$$

which is the well known partition function. When dealing with more than one configuration, the product Gibbs measure is denoted by $\Omega$. Like we just did, we will often omit the dependence on $\beta$ and on the size of the system $N$ of various quantities. In general, we will commit some slight notational abuses to lighten the expression when there is no risk of confusion. The 
free energy density $f_{N}$ is defined by

$$
-\beta f_{N}(\beta)=\frac{1}{N} \mathbb{E} \ln Z_{N}(\beta) .
$$

The whole physical content of the model is carried by [7] the even multioverlaps $q_{1 \cdots 2 n}$, defined by

$$
q_{1 \cdots 2 n}=\frac{1}{N} \sum_{i=1}^{N} \sigma_{i}^{(1)} \cdots \sigma_{i}^{(2 n)} .
$$

\section{Cavity approach and Random Multi-Overlap Structures}

The thermodynamic limit of the free energy density exists if and only if the sequence of the increments (due to the addition of a particle to the system) is convergent in the Cesàro sense (indicated by a boldface $\mathbf{C}$ ):

$$
\lim _{M \rightarrow \infty} \frac{1}{M} \mathbb{E} \ln Z_{M} \equiv \lim _{M \rightarrow \infty} \frac{1}{M} \sum_{n=0}^{M-1} \mathbb{E} \ln \frac{Z_{n+1}}{Z_{n}} \equiv \mathbf{C} \lim _{M \rightarrow \infty} \mathbb{E} \ln \frac{Z_{M+1}}{Z_{M}} .
$$

The idea at the basis of the cavity approach is in fact to measure the effect on the free energy of the addition of one particle to the system (see 4 for a beautiful summary). Let us denote the given $M$ spins by $\tau$, as we want to save the symbol $\sigma$ for the added $\operatorname{spin}(\mathrm{s})$. Now, following [7], we can write, in distribution,

$$
-H_{M+1}(\tau, \sigma ; \alpha) \sim \sum_{\nu=1}^{P_{\alpha \frac{M 2}{M+1}}} J_{\nu} \tau_{k_{\nu}} \tau_{l_{\nu}}+\sum_{\nu=1}^{P_{\alpha} \frac{2 M}{M+1}} \tilde{J}_{\nu} \tau_{k_{\nu}} \sigma_{i_{\nu}},
$$

where we neglected a term which does not contribute when $M$ is large [7, $\left\{\tilde{J}_{\nu}\right\}$ are independent copies of $J,\left\{k_{\nu}\right\}$ and $\left\{l_{\nu}\right\}$ are independent random variables all uniformly distributed over $\{1, \ldots, M\},\left\{i_{\nu}\right\}$ are independent random variables uniformly distributed over the set $\{1, \ldots, N \equiv 1\}$, consisting of $\{1\}$ only. So $\sigma_{i_{\nu}} \equiv \sigma_{1}$. Notice that we can also write, in distribution,

$$
H_{M+1}(\tau, \sigma ; \alpha) \sim H_{M}\left(\tau ; \alpha^{\prime}\right)+\tilde{h}_{\tau} \sigma_{1}
$$


where

$$
\alpha^{\prime}=\alpha(M /(M+1)), \tilde{h}_{\tau}=-\sum_{\nu=1}^{P_{2 \alpha^{\prime}}} \tilde{J}_{\nu} \tau_{k_{\nu}} .
$$

Notice also that similarly

$$
H_{M}(\tau ; \alpha)=H_{M}\left(\tau ; \alpha^{\prime}, \mathcal{J}\right)+H_{M}\left(\tau ; \alpha^{\prime} / M, \hat{\mathcal{J}}\right)
$$

thanks to the additivity property of Poisson variables, and the two Hamiltonians in the right hand side have independent quenched random variables. Hence, if we call

$$
H_{M}\left(\tau ; \alpha^{\prime} / M, \hat{\mathcal{J}}\right)=\hat{H}_{\tau}\left(\alpha^{\prime}\right)=-\sum_{\nu=1}^{P_{\alpha^{\prime}}} \hat{J}_{\nu} \tau_{k_{\nu}} \tau_{l_{\nu}},
$$

then

$$
\mathbb{E} \ln \frac{Z_{M+1}}{Z_{M}}=\mathbb{E} \ln \frac{\sum_{\tau, \sigma} \xi_{\tau} \exp \left(-\beta \tilde{h}_{\tau} \sigma\right)}{\sum_{\tau} \xi_{\tau} \exp \left(-\beta \hat{H}_{\tau}\right)},
$$

with

$$
\xi_{\tau}=\exp \left(-\beta H\left(\tau ; \alpha^{\prime}\right)\right) .
$$

As elegantly explained in 4] (which we quote), this equation expresses the incremental contribution to the free energy in terms of the mean free energy of a particle added to a reservoir whose internal state is described by $\left(\tau, \xi_{\tau}\right)$, corrected by an inverse-fugacity term $\hat{H}$, which encodes a connectivity shift. The latter may be thought of as the free energy of a "place holder": the cavity into which the $(M+1)$ st particle is added. One may note that the addition of a particle to the reservoir of $M$ particles has an effect on the state of the reservoir. For $M>>1$, the value of the added spin, $\sigma$, does not affect significantly the field which would exist for the next increment in $M$. Hence, for the next addition of a particle we may continue to regard the state of the reservoir as given by just the configuration $\tau$. However, the weight of the configuration (which is still to be normalized to yield its probability) undergoes the change:

$$
\xi_{\tau} \rightarrow \xi_{\tau} e^{-\beta \tilde{h}_{\tau} \sigma}
$$


This transformation is called cavity dynamics.

When we add more particles to the system, they do not interact, as there will just be copies of the cavity fields $\tilde{h}^{i}$ acting paramagnetically on each added spin (see [7 for details). Therefore if we add infinitely many particles (to an already infinite reservoir), we can replace the initial complicated model with a (at least in principle) simpler paramagnet. The reasoning just illustrated has thus paved the way to the proper concept to introduce for the computation of the free energy [7].

Definition $1 A$ Random Multi-Overlap Structure $\mathcal{R}$ is a triple $\left(\Sigma,\left\{\tilde{q}_{2 n}\right\}, \xi\right)$ where

- $\Sigma$ is a discrete space;

- $\xi: \Sigma \rightarrow \mathbb{R}_{+}$is a system of random weights;

- $\tilde{q}_{2 n}: \Sigma^{2 n} \rightarrow[0,1], n \in \mathbb{N},|\tilde{q}| \leq 1$ is a positive definite Multi-Overlap Kernel (equal to 1 on the diagonal of $\Sigma^{2 n}$ ).

By looking at the properties of $\tilde{h}, \hat{H}$ in (2)-(3), we know that when many particles ( say $N$ ) are added to the system, we need [7] in general $N+1$ random variables $\left\{\tilde{h}_{\gamma}^{i}(\alpha ; \tilde{J})\right\}_{i=1}^{N}$ and $\hat{H}_{\gamma}(\alpha ; \hat{J})$ such that

$$
\begin{aligned}
\frac{d}{d \alpha} \mathbb{E} \ln \sum_{\gamma} \xi_{\gamma} \exp \left(-\beta \tilde{h}_{i}\right) & =2 \sum_{n>0} \frac{1}{2 n} \mathbb{E} \tanh ^{2 n}(\beta J)\left(1-\left\langle\tilde{q}_{2 n}\right\rangle\right) \\
\frac{d}{d \alpha} \mathbb{E} \ln \sum_{\gamma} \xi_{\gamma} \exp (-\beta \hat{H}) & =\sum_{n>0} \frac{1}{2 n} \mathbb{E} \tanh ^{2 n}(\beta J)\left(1-\left\langle\tilde{q}_{2 n}^{2}\right\rangle\right) .
\end{aligned}
$$

These are the fields to plug into the trial pressure

$$
G_{N}(\mathcal{R})=\frac{1}{N} \mathbb{E} \ln \frac{\sum_{\gamma, \sigma} \xi_{\gamma} \exp \left(-\beta \sum_{i=1}^{N} \tilde{h}_{\gamma}^{i} \sigma_{i}\right)}{\sum_{\gamma} \xi_{\gamma} \exp \left(-\beta \hat{H}_{\gamma}\right)} .
$$

The Boltzmann RaMOSt [7] is the one we started from, constructed by thinking of a reservoir of $M$ spins $\tau$

$$
\Sigma=\{-1,1\}^{M} \ni \tau, \xi_{\tau}=\exp \left(-\beta H_{M}\right), \tilde{q}_{1 \cdots 2 n}=\frac{1}{M} \sum_{k=1}^{M} \tau_{k}^{(1)} \cdots \tau_{k}^{(2 n)}
$$


with

$$
\tilde{h}_{i}(\alpha)=\sum_{\nu=1}^{P_{2 \alpha}} \tilde{J}_{\nu}^{i} \tau_{k_{\nu}^{i}}, \hat{H}(\alpha N)=-\sum_{\nu=1}^{P_{\alpha N}} \hat{J}_{\nu} \tau_{k_{\nu}} \tau_{l_{\nu}}
$$

and $\hat{J}$ independent copies of $J$, independent of any other copy.

The next theorem will not be used, but it justifies the whole machinery described so far.

Theorem 1 (Extended Variational Principle) Infimizing for each $N$ separately the trial function $G_{N}(\mathcal{R})$ over the whole RaMOSt space, the resulting sequence tends to the limiting pressure $-\beta f$ of the $V B$ model as $N$ tends to infinity:

$$
-\beta f=\lim _{N \rightarrow \infty} \inf _{\mathcal{R}} G_{N}(\mathcal{R}) .
$$

A RaMOSt $\mathcal{R}$ is said to be optimal if $G(\mathcal{R})=-\beta f(\beta) \forall \beta$. We will denote by $\Omega$ the measure associated to the RaMOSt weights $\xi$ as well.

Let $c_{i}=2 \cosh \left(\beta \tilde{h}^{i}\right)$. Is it possible to show [7] that optimal RaMOSt's enjoy the same factorization property enjoyed by the Boltzmann RaMOSt and described in the next

Theorem 2 (Factorization of optimal RaMOSt's) In the whole region where the parameters are uniquely defined, the following Cesàro limit is linear in $N$ and $\bar{\alpha}$

$$
\mathbf{C} \lim _{M} \mathbb{E} \ln \Omega_{M}\left\{c_{1} \cdots c_{N} \exp [-\beta \hat{H}(\bar{\alpha})]\right\}=N(-\beta f+\alpha A)+\bar{\alpha} A,
$$

where

$$
A=\sum_{n=1}^{\infty} \frac{1}{2 n} \mathbb{E} \tanh ^{2 n}(\beta J)\left(1-\left\langle q_{2 n}^{2}\right\rangle\right) .
$$

We will use only part of this theorem, or more precisely a modification of the part involving the inverse fugacity only. Notice that in the definition (6) of trial pressure $G$ the part with the cavity fields and the part with the inverse fugacity are taken already factorized. If we therefore focus on the fugacity part only in the theorem above, by putting all the cavity fields 
$\tilde{h}^{i}$ to zero, the property described becomes what is often called stochastic stability (of the measure $\Omega$ with respect to the perturbation $\hat{H}$ ), which we will prove and use in Section 5 It should be now clear, from the construction described about equations (22)-(3), that this perturbation can be either due to the addition of $N$ particles, or due to a connectivity shift, and leads to a linear response of the free energy. Hence the theorem above merges two invariance properties of the optimal RaMOSt measure $\Omega$ : the one of the cavity part with respect to the cavity dynamics, and the one of the fugacity part with respect to the connectivity shifts. The invariance with respect to the cavity dynamics is a special case of Quasi-Stationarity, i.e. the invariance up to a correcting factor under random shifts (see [3, 4] for a proper introduction). The Parisi Ultrametric Ansatz, both for dilute and for fully connected Gaussian models [15, is based on Hierarchical Random Probability Cascades, which exhibits the Quasi-Stationarity of the Generalized Random Energy Model [4, 15. A very stimulating conjecture is that Random Probability Cascades include all the quasi-stationary structures.

As a side remark, we point out that for stochastically stable systems, a dynamical order parameter can be defined and related to the static order parameter, and that ultrametricity in the dynamics implies static ultrametricity, which is in turn implied by the so-called separability, and connected to the overalp equivalence. We refer to [11, 14, 9] for details, in general we just stress that all the concepts introduced in these last comments, and the one of self-averaging, are intimately related, and have deep physical meanings.

\section{Non-negativity of the average of multi-overlaps}

We know from [8, 13] that above the critical temperature $\beta_{c}$ all the multioverlaps (including the 2-overlap) are identically zero, as the replica symmetric solution holds. We also know, from [13, that the (rescaled) 2-overlap shows diverging fluctuations at the critical temperature $\beta_{c}$ where the replica 
symmetry is broken, while the (rescaled) multi-overlap of more than two replicas do not exhibit diverging fluctuations at this inverse temperature. The fluctuations of the (rescaled) multi-overlaps do diverge at lower critical temperatures. The critical temperature $\beta_{c}^{(2 n)}$ at which the fluctuations of $\sqrt{N} q_{2 n}$ diverge is defined by

$$
\tanh ^{2 n}\left(\beta_{c}^{(2 n)}\right)=\frac{1}{2 \alpha}, \alpha>\frac{1}{2} .
$$

We want to show that all the multi-overlap exhibits the same phase transition as the 2-overlap in the Gaussian SK model [1]: if we apply an external field and then remove it in the thermodynamic limit, each multi-overlap remains strictly positive below its critical temperature, where its variance becomes non-zero.

Let us introduce the following notation: $\omega_{\bar{\alpha}}(\cdot),\langle\cdot\rangle_{\bar{\alpha}}$ denote the usual expectations except for a perturbation in the Boltzmannfaktor, which is assumed here to be

$$
\exp \left[-\beta\left(H_{N}(\sigma ; \alpha)-\sum_{i=1}^{N}\left(\sum_{\nu=1}^{P_{\bar{\alpha}}^{i}} \tilde{J}_{\nu}^{i}\right) \sigma_{i}\right)\right],
$$

i.e. the initial Boltzmannfaktor is perturbed with independent copies of an external field $\tilde{h}(\bar{\alpha})=\sum_{\nu=1}^{P_{\bar{\alpha}}} \tilde{J}_{\nu}$ modulated by $\bar{\alpha}$.

This section is devoted to the next

\section{Theorem 3 The following holds}

1. for any integer $n>0$ and any inverse temperature $\beta$

$$
\lim _{N \rightarrow \infty}\left\langle q_{1 \cdots 2 n}\right\rangle_{\bar{\alpha}=2 \alpha / N} \geq 0
$$

2. for any integer $n>0$ and for any $\beta>\beta_{c}^{(2 n)}$, defined by

$$
2 \alpha \tanh ^{2 n}\left(\beta_{c}^{(2 n)}\right)=1 \text {, }
$$

$$
\lim _{N \rightarrow \infty}\left\langle q_{12}\right\rangle_{\bar{\alpha}=2 \alpha / N}>0
$$


The theorem will be a simple consequence of a couple of lemmas, which require a definition as well.

Lemma 1 Consider the set of indices $\left\{i_{1}, . ., i_{r}\right\}$, with $r \in[1, N]$. Then

$$
\lim _{\bar{\alpha} \rightarrow 2 \alpha / N} \omega_{N, \bar{\alpha}}\left(\sigma_{i_{1}}, \ldots, \sigma_{i_{r}}\right)=\omega_{N+1}\left(\sigma_{i_{1}}, \ldots, \sigma_{i_{r}}, \sigma_{N+1}^{r}\right)+O\left(\frac{1}{N}\right),
$$

where $r$ is an exponent and we made explicit the dependence of $\omega$ on the size of the system.

This lemma is a consequence of the fact that with the chosen $\bar{\alpha}$ the presence of the external field is equivalent to the introduction of an additional particle, labeled $N+1$. This should be clear from (11)-(2) and from the gauge symmetry with respect to the transformation $\sigma_{i} \rightarrow \sigma_{i} \sigma_{N+1}$, but the reader may want to look at [7, 1, for details. We are not interested here in the fullest generality, and we will limit ourselves to the following

Definition 2 A polynomial function of some overlaps is said

- filled if every replica appears an even number of times in it;

- fillable if it can be made filled multiplying it by exactly one multioverlap of properly chosen replicas;

The next lemma is a straightforward generalization of the analogous one proven in [5], and relies on the fact that for filled polynomial the exponent $r$ is even.

Lemma 2 In the $N \rightarrow \infty$ limit the average $\langle\cdot\rangle_{\bar{\alpha}}$ of filled polynomials is not affected by the presence of the perturbation modulated by $\bar{\alpha}$, that is, for instance, $\left\langle q_{12} q_{23} q_{13}\right\rangle_{\bar{\alpha}}=\left\langle q_{12} q_{23} q_{13}\right\rangle$, with the possible exclusion of a zero Lebesgue measure set of values of $\bar{\alpha}$.

From this lemma we will deduce the next 
Proposition 1 Let $Q_{1 \cdots 2 n}$ be a fillable polynomial of the overlaps, i.e. $q_{1 \cdots 2 n} Q_{1 \cdots 2 n}$ is filled. Then

$$
\lim _{N \rightarrow \infty} \lim _{\bar{\alpha} \rightarrow 2 \alpha / N}\left\langle Q_{1 \cdots 2 n}\right\rangle_{\bar{\alpha}}=\left\langle q_{1 \cdots 2 n} Q_{1 \cdots 2 n}\right\rangle,
$$

where the right hand side is understood to be taken in the thermodynamic limit too.

Proof. A fillable polynomial will be taken of the form

$$
Q_{1 \cdots 2 n}=\sum_{i_{1} \cdots i_{2 n}} \frac{\sigma_{i_{1}}^{1} \cdots \sigma_{i_{2 n}}^{2 n}}{N^{2 n}} \tilde{Q}_{i_{1} \cdots i_{2 n}}(\sigma)
$$

so that the $1, \ldots, 2 n$ label the non-filled replicas, fillable by $q_{1 \cdots 2 n}$, and the filled replicas are included in $\tilde{Q}_{i_{1} \cdots i_{2 n}}$. We have

$$
\begin{aligned}
\left\langle Q_{1 \cdots 2 n}\right\rangle_{\bar{\alpha}}=\frac{1}{N^{2 n}} \mathbb{E} \sum_{i_{1} \cdots i_{2 n}} \Omega_{\bar{\alpha}}\left(\sigma_{i_{1}}^{1} \cdots \sigma_{i_{2 n}}^{2 n} \tilde{Q}_{i_{1} \cdots i_{2 n}}(\sigma)\right)= \\
\frac{1}{N^{2 n}} \mathbb{E} \sum_{i_{1} \cdots i_{2 n}} \omega_{\bar{\alpha}}\left(\sigma_{i_{1}}^{1}\right) \cdots \omega_{\bar{\alpha}}\left(\sigma_{i_{2 n}}^{2 n}\right) \Omega_{\bar{\alpha}}\left(\tilde{Q}_{i_{1} \cdots i_{2 n}}\right) .
\end{aligned}
$$

We will now take $\bar{\alpha}=2 \alpha / N$ and use Lemma 1 to get

$$
\omega_{\bar{\alpha}=2 \alpha / N}\left(\sigma_{i}^{a}\right)=\omega\left(\sigma_{i}^{a} \sigma_{N+1}^{a}\right)+O\left(\frac{1}{N}\right)
$$

while as $\tilde{Q}$ is concerned the product state $\Omega_{\bar{\alpha}}$ continues to act on an even number of each replica and it is not modified, thanks to Lemma 2

$$
\Omega_{\bar{\alpha}=2 \alpha / N}\left(\tilde{Q}_{i_{1} \cdots i_{2 n}}\right)=\Omega\left(\tilde{Q}_{i_{1} \cdots i_{2 n}}\right) .
$$

Hence

$\omega\left(\sigma_{i}^{1} \sigma_{N+1}^{1}\right) \cdots \omega\left(\sigma_{i}^{2 n} \sigma_{N+1}^{2 n}\right) \Omega\left(\tilde{Q}_{i_{1} \cdots i_{2 n}}\right)=\Omega\left(\sigma_{i_{1}}^{1} \cdots \sigma_{i_{2 n}}^{2 n} \sigma_{N+1}^{1} \cdots \sigma_{N+1}^{2 n} \tilde{Q}_{i_{1} \cdots i_{2 n}}\right)$.

Using the dummy summation variable $\sigma_{N+1}$, 1] we can also sum over all dumb indexes from 1 to $N$ and divide by $N$, because the involved terms are of order $O\left(\frac{1}{N}\right)$ and become irrelevant in the $N \rightarrow \infty$ limit. Therefore $\left\langle Q_{1 \cdots 2 n}\right\rangle_{\bar{\alpha}=2 \alpha / N}=N^{-2 n-1} \mathbb{E} \sum_{i_{1} \cdots i_{2 n} j} \Omega\left(\sigma_{j}^{1} \cdots \sigma_{j}^{2 n} \sigma_{i_{1}}^{1} \cdots \sigma_{i_{2 n}}^{2 n}\right) \tilde{Q}_{i_{1} \cdots i_{2 n}}+O\left(\frac{1}{N}\right)$ 
and in the thermodynamic limit the proof is complete.

At this point Theorem 3 is simply a corollary of this proposition in the case $Q=q$.

\section{Stability relations from Quasi-Stationarity}

In this section we want to prove the following

Theorem 4 The consequences of stochastic stability in fully connected models extend to dilute spin glasses, and constraints, analogous to those found for overlaps of two replicas only, hold for multi-overlaps. More precisely,

1. the Aizenman-Contucci relations hold in dilute spin glasses. A first example is

$$
\left\langle q_{12}^{2} q_{13}^{2}\right\rangle=\frac{1}{4}\left\langle q_{12}^{4}\right\rangle+\frac{3}{4}\left\langle q_{12}^{2} q_{34}^{2}\right\rangle ;
$$

2. further relations for multi-overlaps hold in dilute spin glasses. A first example is

$$
\left\langle q_{1234}^{2} q_{15}^{2}\right\rangle=\frac{3}{8}\left\langle q_{1234}^{2} q_{12}^{2}\right\rangle+\frac{5}{8}\left\langle q_{1234}^{2} q_{56}^{2}\right\rangle
$$

We start addressing the proof of the theorem by proving a lemma that gives the explicit form of the contribution to the free energy of a connectivity shift.

Lemma 3 Let $\Omega,\langle\cdot\rangle$ be the usual Gibbs and quenched Gibbs expectations at inverse temperature $\beta$, associated to the Hamiltonian $H_{N}(\sigma, \alpha ; \mathcal{J})$. Then, in the whole region where the parameters are uniquely defined

$$
\lim _{N \rightarrow \infty} \mathbb{E} \ln \Omega \exp \left(\beta^{\prime} \sum_{\nu=1}^{P_{\alpha^{\prime}}} J_{\nu}^{\prime} \sigma_{i_{\nu}^{\prime}} \sigma_{j_{\nu}^{\prime}}\right)=\alpha^{\prime} \sum_{n=1}^{\infty} \frac{1}{2 n} \tanh ^{2 n}\left(\beta^{\prime}\right)\left(1-\left\langle q_{2 n}^{2}\right\rangle\right),
$$

where the random variables $P_{\alpha^{\prime}},\left\{J_{\nu}^{\prime}\right\},\left\{i_{\nu}^{\prime}\right\},\left\{j_{\nu}^{\prime}\right\}$ are independent copies of the homologous ones appearing in the Hamiltonian in $\Omega$. 
Notice that, in distribution

$$
\beta \sum_{\nu=1}^{P_{\alpha N}} J_{\nu} \sigma_{i_{\nu}} \sigma_{j_{\nu}}+\beta^{\prime} \sum_{\nu=1}^{P_{\alpha^{\prime}}} J_{\nu}^{\prime} \sigma_{i_{\nu}^{\prime}} \sigma_{j_{\nu}^{\prime}} \sim \beta \sum_{\nu=1}^{P_{\left(\alpha+\alpha^{\prime} / N\right) N}} J_{\nu}^{\prime \prime} \sigma_{i_{\nu}} \sigma_{j_{\nu}}
$$

where $\left\{J_{\nu}^{\prime \prime}\right\}$ are independent copies of $J$ with probability $\alpha N /\left(\alpha N+\alpha^{\prime}\right)$ and independent copies of $J \beta^{\prime} / \beta$ with probability $\alpha^{\prime} /\left(\alpha N+\alpha^{\prime}\right)$. In the right hand side above, the quenched random variables will be collectively denoted by $\mathcal{J}^{\prime \prime}$. Notice also that, for this reason, and since the sum of Poisson random variables is a Poisson random variable with mean equal to the sum of the means, we have

$$
A_{t} \equiv \mathbb{E} \ln \Omega \exp \left(\beta^{\prime} \sum_{\nu=1}^{P_{\alpha^{\prime} t}} J_{\nu}^{\prime} \sigma_{i_{\nu}^{\prime}} \sigma_{j_{\nu}^{\prime}}\right)=\mathbb{E} \ln \frac{Z_{N}\left(\alpha_{t} ; \mathcal{J}^{\prime \prime}\right)}{Z_{N}(\alpha ; \mathcal{J})}
$$

where we defined, for $t \in[0,1]$,

$$
\alpha_{t}=\alpha+\alpha^{\prime} \frac{t}{N}
$$

so that $\alpha_{t} \rightarrow \alpha \forall t$ as $N \rightarrow \infty$, and $\alpha_{0}=\alpha, \alpha_{1} \equiv \alpha^{\prime \prime}$.

Proof. Let us compute the $t$-derivative of $A_{t}$, just defined in (11)

$$
\frac{d}{d t} A_{t}=\mathbb{E} \sum_{m=1}^{\infty} \frac{d}{d t} \pi_{\alpha^{\prime} t}(m) \ln \sum_{\sigma} \exp \left(\beta^{\prime} \sum_{\nu=1}^{m} J_{\nu}^{\prime} \sigma_{i_{\nu}^{\prime}} \sigma_{j_{\nu}^{\prime}}\right) .
$$

Using the following elementary property of the Poisson measure

$$
\frac{d}{d t} \pi_{t \zeta}(m)=\zeta\left(\pi_{t \zeta}(m-1)-\pi_{t \zeta}(m)\right)
$$

we get

$$
\begin{aligned}
& \frac{d}{d t} A_{t}= \alpha^{\prime} \mathbb{E} \sum_{m=0}^{\infty}\left[\pi_{\alpha^{\prime} t}(m-1)-\pi_{\alpha^{\prime} t}(m)\right] \ln \sum_{\sigma} \exp \left(\beta^{\prime} \sum_{\nu=1}^{m} J_{\nu}^{\prime} \sigma_{i_{\nu}^{\prime}} \sigma_{j_{\nu}^{\prime}}\right) \\
&= \alpha^{\prime} \mathbb{E} \ln \sum_{\sigma} \exp \left(\beta^{\prime} J^{\prime} \sigma_{i_{\nu}^{\prime}} \sigma_{j_{\nu}^{\prime}}\right) \exp \left(\beta^{\prime} \sum_{\nu=1}^{P_{\alpha^{\prime} t}} J_{\nu}^{\prime} \sigma_{i_{\nu}^{\prime}} \sigma_{j_{\nu}^{\prime}}\right) \\
&-\alpha^{\prime} \mathbb{E} \ln \sum_{\sigma} \exp \left(\beta^{\prime} \sum_{\nu=1}^{P_{\alpha^{\prime} t}} J_{\nu}^{\prime} \sigma_{i_{\nu}^{\prime}} \sigma_{j_{\nu}^{\prime}}\right) \\
&=\alpha^{\prime} \mathbb{E} \ln \Omega_{t} \exp \left(\beta^{\prime} J^{\prime} \sigma_{i_{\nu}^{\prime}} \sigma_{j_{\nu}^{\prime}}\right),
\end{aligned}
$$


where we included the $t$-dependent weights in the average $\Omega_{t}$. Now use the following identity

$$
\exp \left(\beta^{\prime} J^{\prime} \sigma_{i} \sigma_{j}\right)=\cosh \left(\beta^{\prime} J^{\prime}\right)+\sigma_{i} \sigma_{j} \sinh \left(\beta^{\prime} J^{\prime}\right)
$$

to get

$$
\frac{d}{d t} A_{t}=\alpha^{\prime} \mathbb{E} \ln \Omega_{t}\left[\cosh \left(\beta^{\prime} J^{\prime}\right)\left(1+\tanh \left(\beta^{\prime} J^{\prime}\right) \sigma_{i_{\nu}^{\prime}} \sigma_{j_{\nu}^{\prime}}\right)\right] .
$$

It is clear that

$$
\mathbb{E} \omega_{t}^{2 n}\left(\sigma_{i_{\nu}} \sigma_{j_{\nu}}\right)=\left\langle q_{2 n}^{2}\right\rangle_{t},
$$

so we now expand the logarithm in power series, and we see that, in the limit of large $N$, as $\alpha_{t} \rightarrow \alpha$ the result does not depend on $t$, everywhere the measure $\langle\cdot\rangle_{t}$ is continuous as a function of the parameter $t$. Thanks to the comments that preceded the current proof, formalized in (9)-(10)-(11), this is the same as assuming that $\Omega$ is regular as a function of $\alpha$, because $J^{\prime \prime} \rightarrow J$ in the sense that in the large $N$ limit $J^{\prime \prime}$ can only take the usual values \pm 1 since the probability of being $\pm \beta^{\prime} / \beta$ becomes zero. Therefore integrating back against $t$ from 0 to 1 is the same as multiplying by 1 . Thanks to the symmetric distribution of $J$, the expansion of the logarithm yields the result, where the odd powers are missing.

Remark 1 The same result holds for the hierarchical Parisi trial structure [8]. In general, what we study in this section relies only on (8), that holds for quasi-stationary RaMOSt's, to which our results therefore extend.

Proof of Theorem 4. Consider once again

$$
\hat{H}=-\sum_{\nu=1}^{P_{\alpha^{\prime}}} J_{\nu}^{\prime} \sigma_{i_{\nu}^{\prime}} \sigma_{j_{\nu}^{\prime}} .
$$

We will let again $\Omega$ be the infinite volume Gibbs measure associated to the VB Hamiltonian at connectivity $\alpha$ and inverse temperature $\beta$.

By the assumed symmetry of $\Omega$, we have $[\underline{6}$

$$
\mathbb{E} \ln \Omega \exp \left(\beta^{\prime} \hat{H}\right)=\frac{1}{2} \mathbb{E} \ln \Omega \exp \left(-\beta^{\prime}\left(\hat{H}-\hat{H}^{\prime}\right)\right),
$$


where we replicated $\hat{H}^{\prime}=\hat{H}\left(\sigma^{\prime}\right)$.

For the left hand side of (8), a tedious expansion yields

$$
\begin{aligned}
& \frac{1}{2} \ln \Omega \exp \left(-\beta^{\prime}\left(\hat{H}-\hat{H}^{\prime}\right)\right)= \\
& \beta^{\prime 2} \frac{1}{4}\left[2 \Omega\left(\hat{H}^{2}\right)-2 \Omega^{2}(\hat{H})\right]+ \\
& \beta^{\prime 4} \frac{1}{24}\left[\Omega\left(\hat{H}^{4}\right)-4 \Omega\left(\hat{H}^{3}\right) \Omega(\hat{H})-3 \Omega^{2}\left(\hat{H}^{2}\right)+12 \Omega\left(\hat{H}^{2}\right) \Omega^{2}(\hat{H})-6 \Omega^{4}(\hat{H})\right]+ \\
& \beta^{\prime 6}\left[\frac{1}{6 !} \Omega\left(\hat{H}^{6}\right)-\frac{1}{5 !} \Omega\left(\hat{H}^{5}\right) \Omega(\hat{H})-\frac{1}{48} \Omega\left(\hat{H}^{4}\right) \Omega\left(\hat{H}^{2}\right)-\frac{1}{72} \Omega^{2}\left(\hat{H}^{3}\right)\right. \\
& \quad+\frac{1}{6} \Omega(\hat{H}) \Omega\left(\hat{H}^{2}\right) \Omega\left(\hat{H}^{3}\right)+\frac{1}{24} \Omega^{3}\left(\hat{H}^{2}\right)+\frac{1}{24} \Omega^{2}(\hat{H}) \Omega\left(\hat{H}^{4}\right)-\frac{1}{6} \Omega\left(\hat{H}^{3}\right) \Omega^{3}(\hat{H}) \\
& \left.\quad-\frac{3}{8} \Omega^{2}(\hat{H}) \Omega^{2}\left(\hat{H}^{2}\right)+\frac{1}{2} \Omega\left(\hat{H}^{2}\right) \Omega^{4}(\hat{H})-\frac{1}{6} \Omega^{6}(\hat{H})\right]+O\left(\beta^{\prime 8}\right),
\end{aligned}
$$

of which we have to take the quenched expectation $\mathbb{E}$, using the formulas in Appendix $\mathrm{A}$. While for the right hand side of (8), we have, from the expansion of the hyperbolic tangent reported for convenience in Appendix A

$$
\begin{aligned}
\alpha^{\prime} \sum_{n>0} \frac{1}{2 n} \tanh ^{2 n}\left(\beta^{\prime}\right) & \left(1-\left\langle q_{1 \cdots 2 n}^{2}\right\rangle\right)= \\
& \beta^{\prime 2} \alpha^{\prime}\left(\frac{1}{2}-\frac{1}{2}\left\langle q_{12}^{2}\right\rangle\right)+ \\
& \beta^{\prime 4} \alpha^{\prime}\left(-\frac{1}{12}+\frac{1}{3}\left\langle q_{12}^{2}\right\rangle-\frac{1}{4}\left\langle q_{1234}^{2}\right\rangle\right)+ \\
& \beta^{\prime 6} \alpha^{\prime}\left(\frac{2}{90}-\frac{17}{90}\left\langle q_{12}^{2}\right\rangle+\frac{1}{3}\left\langle q_{1234}^{2}\right\rangle-\frac{1}{6}\left\langle q_{123456}^{2}\right\rangle\right)+O\left(\beta^{\prime 8}\right)
\end{aligned}
$$

Recall that the averages $\langle\cdot\rangle$ do not depend on $\beta^{\prime}$, so we have to power series in $\beta^{\prime}$ that we can equate term by term. The order zero and the odd orders are absent in both sides. Let us consider the second order. Taking into account the formulas given in Appendix A, we have two identical (constant) monomials in $\alpha^{\prime}$

$$
\frac{1}{2}\left(1-\left\langle q_{12}^{2}\right\rangle\right)=\frac{1}{2}\left(1-\left\langle q_{12}^{2}\right\rangle\right)
$$

and we gain no information. Let us move on to order four: using again the formulas given in Appendix $\mathrm{A}$, we have the equality of two polynomials of 
degree two in $\alpha^{\prime}$. There is no constant term, and no second power in the right hand side. Equating term by term we get an trivial identity for the linear part in $\alpha^{\prime}$ (and hence no information). We get instead the following relation from the quadratic term in $\alpha^{\prime}$

$$
\left\langle q_{12}^{2} q_{13}^{2}\right\rangle=\frac{1}{4}\left\langle q_{12}^{4}\right\rangle+\frac{3}{4}\left\langle q_{12}^{2} q_{34}^{2}\right\rangle
$$

which is the first AC relation, that we wanted to prove.

Notice that in the linear part in $\alpha^{\prime}$ a multi-overlap is present: $q_{1234}$, but it gets canceled out. In the quadratic part, instead, only 2-overlaps are present.

Let us now focus on the sixth order. Again we get a useless identity from the linear part in $\alpha^{\prime}$, from which 4-overlaps and 6-overlaps get canceled out, and we gain no information. From the quadratic part, where 4-overlaps are present (but no 6-overlaps are there), $\left\langle q_{12}^{2}\right\rangle$ disappears, and the other terms with 2-overlaps only get canceled out thanks to the previous AC relation. In fact, from the expansion we have (we provide the result of the direct calculation without skipping many simplification, for easier verification)

$$
\begin{aligned}
& \frac{15}{6 !}- \frac{15}{5 !}\left\langle q_{12}^{2}\right\rangle-\frac{7}{48}-\frac{1}{6}\left\langle q_{12}^{4}\right\rangle-\frac{15}{72}\left\langle q_{12}^{2}\right\rangle+\frac{7}{6}\left\langle q_{12}^{2}\right\rangle+ \\
& \frac{4}{3}\left\langle q_{12}^{2} q_{23}^{2}\right\rangle+\frac{1}{8}+\frac{1}{2}\left\langle q_{12}^{4}\right\rangle+\frac{1}{24}\left\langle q_{12}^{2}\right\rangle+\frac{1}{3}\left\langle q_{12}^{2} q_{23}^{2}\right\rangle+\frac{1}{4}\left\langle q_{12}^{2}\right\rangle- \\
& \frac{1}{2}\left\langle q_{1234}^{2}\right\rangle-2\left\langle q_{12}^{2} q_{34}^{2}\right\rangle-\frac{9}{8}\left\langle q_{12}^{2}\right\rangle-3\left\langle q_{12}^{2} q_{23}^{2}\right\rangle-\frac{3}{2}\left\langle q_{12}^{2} q_{1234}^{2}\right\rangle+ \\
& \frac{1}{2}\left\langle q_{1234}^{2}\right\rangle+3\left\langle q_{12}^{2} q_{34}^{2}\right\rangle+4\left\langle q_{12}^{2} q_{2345}^{2}\right\rangle-\frac{15}{6}\left\langle q_{12}^{2} q_{3456}^{2}\right\rangle=0 .
\end{aligned}
$$

So we are left, after a few trivial calculations, with the following new relation for multi-overlaps

$$
\left\langle q_{1234}^{2} q_{15}^{2}\right\rangle=\frac{3}{8}\left\langle q_{1234}^{2} q_{12}^{2}\right\rangle+\frac{5}{8}\left\langle q_{1234}^{2} q_{56}^{2}\right\rangle
$$

announced in the statement of the theorem.

In the cubic part only overlaps of two replicas are present, and only the monomials of order six remain, as the lower degree ones cancel out directly. 
The remaining relation is

$$
\begin{aligned}
\frac{1}{12}\left\langle q_{12}^{6}\right\rangle- & \left\langle q_{12}^{2} q_{23}^{4}\right\rangle+\frac{3}{4}\left\langle q_{12}^{2} q_{34}^{4}\right\rangle-\frac{1}{3}\left\langle q_{12}^{2} q_{23}^{2} q_{31}^{2}\right\rangle+ \\
& \left\langle q_{12}^{2} q_{13}^{2} q_{14}^{2}\right\rangle+3\left\langle q_{12}^{2} q_{23}^{2} q_{34}^{2}\right\rangle-6\left\langle q_{12}^{2} q_{23}^{2} q_{45}^{2}\right\rangle+\frac{5}{2}\left\langle q_{12}^{2} q_{34}^{2} q_{56}^{2}\right\rangle=0 .
\end{aligned}
$$

Proceeding further, the expansion generates all the identities due to stochastic stability, in full agreement with the self-averaging identities found in [10, 9.

We will provide a more general and systematic form of the relations in the next section.

\section{Stability relations from the cavity stream- ing equation}

In this section we study a family of constraints on the distribution of the overlaps. To address this task we will consider the quenched expectation of a generic function of $s$ replicas, with respect to the perturbed measure with weights

$$
\exp \left(-\beta H_{N}(\sigma ; \alpha)+\beta^{\prime} \sum_{\nu=1}^{P_{2 \alpha^{\prime} t}} \tilde{J}_{\nu} \sigma_{i_{\nu}}\right),
$$

whose use will be indicated with a sub $t$ in the expectations. Once again, $\left\{\tilde{J}_{\nu}\right\}$ are independent copies of $J$.

From now on let us put $\theta=\tanh \left(\beta^{\prime}\right)$, and, assuming $J= \pm 1$, we have $\theta^{2 n}=\mathbb{E} \tanh ^{2 n}\left(\beta^{\prime} J\right), \tanh ^{2 n+1}\left(\beta^{\prime} J\right)=J \theta^{2 n+1} \forall n \in \mathbb{N}$. Let us also just put $\omega_{t}=\omega_{t}(\sigma)$, with a slight abuse of notation.

Proposition 2 Let $\Phi$ be a function of s replicas. Then the following cavity 
streaming equation holds

$$
\begin{array}{r}
\frac{d\langle\Phi\rangle_{t}}{d t}=-2 \alpha^{\prime}\langle\Phi\rangle_{t}+2 \alpha^{\prime} \mathbb{E}\left[\Omega _ { t } \Phi \left\{1+J \sum_{a}^{1, s} \sigma^{a} \theta+\sum_{a<b}^{1, s} \sigma^{a} \sigma^{b} \theta^{2}+\right.\right. \\
\left.J \sum_{a<b<c}^{1, s} \sigma^{a} \sigma^{b} \sigma^{c} \theta^{3}+\cdots\right\}\left\{1-s J \theta \omega_{t}+\frac{s(s+1)}{2 !} \theta^{2} \omega_{t}^{2}-\right. \\
\left.\left.-\frac{s(s+1)(s+2)}{3 !} J \theta^{3} \omega_{t}^{3}+\cdots\right\}\right] \quad \forall \theta
\end{array}
$$

Proof. Let us explicitly perform the calculation of the derivative, using (12).

$$
\begin{array}{r}
\frac{d}{d t} \mathbb{E} Z_{t}^{-1} \sum_{\sigma} \Phi \exp \left(\sum_{a=1}^{s}\left(\beta \sum_{\nu}^{P_{\alpha^{\prime} N}} J_{\nu} \sigma_{i_{\nu}}^{a} \sigma_{j_{\nu}}^{a}+\beta^{\prime} \sum_{\nu}^{P_{2 \alpha^{\prime} t}} \tilde{J}_{\nu} \sigma_{i_{\nu}}^{a}\right)\right)= \\
2 \alpha^{\prime} \mathbb{E} \frac{\Omega_{t}\left[\Phi \exp \left(\beta^{\prime} \sum_{a=1}^{s} J \sigma_{i_{1}}^{a}\right)\right]}{\Omega_{t} \exp \left(\beta^{\prime} \sum_{a=1}^{s} J \sigma_{i_{1}}^{a}\right)}-2 \alpha^{\prime}\langle\Phi\rangle_{t}= \\
2 \alpha^{\prime} \mathbb{E} \frac{\Omega_{t}\left[\Phi \prod_{a=1}^{s}\left(\cosh \left(\beta^{\prime} J\right)+\sigma^{a} \sinh \left(\beta^{\prime} J\right)\right)\right]}{\Omega_{t}\left[\prod_{a=1}^{s}\left(\cosh \left(\beta^{\prime} J\right)+\sigma^{a} \sinh \left(\beta^{\prime} J\right)\right)\right]}-2 \alpha^{\prime}\langle\Phi\rangle_{t}= \\
2 \alpha^{\prime} \mathbb{E} \frac{\Omega_{t}\left[\Phi \prod_{a}^{s}\left(1+J \theta \sigma^{a}\right)\right]}{(1+\theta \omega)^{s}}-2 \alpha^{\prime}\langle\Phi\rangle_{t} .
\end{array}
$$

Now note that

$$
\begin{aligned}
\frac{1}{\left(1+J \theta \omega_{t}\right)^{s}} & =1-J s \theta \omega_{t}+\frac{s(s+1)}{2 !} \theta^{2} \omega_{t}^{2}- \\
& J \frac{s(s+1)(s+2)}{3 !} \theta^{3} \omega_{t}^{3}+\frac{s(s+1)(s+2)(s+3)}{4 !} \theta^{4} \omega_{t}^{4}-\cdots
\end{aligned}
$$

and that

$$
\prod_{a=1}^{s}\left(1+J \theta \sigma_{i_{1}}^{a}\right)=1+J \sum_{a}^{1, s} \sigma_{i_{1}}^{a} \theta+\sum_{a<b}^{1, s} \sigma_{i_{1}}^{a} \sigma_{i_{1}}^{b} \theta^{2}+J \sum_{a<b<c}^{1, s} \sigma_{i_{1}}^{a} \sigma_{i_{1}}^{b} \sigma_{i_{1}}^{c} \theta^{3}+\cdots .
$$

The theorem follows immediately.

In the limit $\alpha^{\prime} \rightarrow \infty$ as $\beta^{\prime}=2 \alpha \theta^{2}$ is kept constant, the powers of $\theta$ higher than two are killed, and we recover the equation for the Gaussian SK model [ 6 ]

$$
\frac{d\langle\Phi\rangle_{t}}{d t}=\left\langle\Phi\left(\sum_{a<b}^{1, s} q_{a b}-s \sum_{a}^{1, s} q_{a, s+1}+\frac{s(s+1)}{2} q_{s+1, s+2}\right)\right\rangle_{t} .
$$


If in the previous theorem we take a function $\Phi$ that does not depend on $t$, the left hand side of (17) is zero, and we have a polynomial in $\theta$ (and hence in $\beta^{\prime}$ ) in the right hand side, that we can equate to zero term by term (we do not need to re-expand in $\beta^{\prime}$ and equate the new coefficients to zero). We know from Lemma 2 that the left hand side of (17) does not depend on $t$ when, for instance, $\Phi$ is a filled polynomial. If, in each term of the expansion that we equate to zero in this case, we additionally take $t=1, \alpha^{\prime}=\alpha, \beta^{\prime}=\beta$, and let $N \rightarrow \infty$, then we also guarantee that the fillable polynomials get filled, thanks to Proposition 1 In other words

Proposition 3 The generator of the constraints on the distribution of the overlap is:

$$
\lim _{N \rightarrow \infty} \partial_{t}\langle\phi\rangle_{t}=0
$$

where $\phi$ is filled, and $t=1, \alpha^{\prime}=\alpha, \beta^{\prime}=\beta$.

Let us consider the first simple example, $\phi=q_{12}^{2}$. Proposition 2 then yields

$$
\begin{array}{r}
\lim _{N \rightarrow \infty} \partial_{t}\left\langle q_{12}^{2}\right\rangle_{t}=\lim _{N \rightarrow \infty}\left\langle q_{12}^{3}-4 q_{12}^{2} q_{23}+3 q_{12}^{2} q_{34}\right\rangle_{t} \theta^{2}+O\left(\theta^{4}\right)= \\
\lim _{N \rightarrow \infty}\left\langle q_{12}^{3}-4 q_{12}^{2} q_{23}+3 q_{12}^{2} q_{34}\right\rangle_{t} \beta^{\prime 2}+O\left(\beta^{\prime 4}\right)=0 \\
\Rightarrow \quad\left\langle q_{12}^{4}-4 q_{12}^{2} q_{23}^{2}+3 q_{12}^{2} q_{34}^{2}\right\rangle=0
\end{array}
$$

which is understood to be taken in the thermodynamic limit and is the same Aizenman-Contucci relation we already found in the previous section.

If we instead choose $\phi=q_{1234}^{2}$, we obtain

$$
\begin{gathered}
\lim _{N \rightarrow \infty} \partial_{t}\left\langle q_{1234}^{2}\right\rangle_{t}=\left\langle\theta^{2}\left(3 q_{1234}^{2} q_{12}^{2}-8 q_{1234}^{2} q_{15}^{2}+10 q_{1234}^{2} q_{152}\right)+\right. \\
\left.\theta^{4}\left(q_{1234}^{4}-16 q_{1234}^{2} q_{1235}^{2}+60 q_{1234}^{2} q_{1256}^{2}-80 q_{1234}^{2} q_{1567}^{2}+35 q_{1234}^{2} q_{5678}^{2}\right)\right\rangle+O\left(\theta^{6}\right)=0
\end{gathered}
$$

From the order two in $\beta^{\prime}$ we have the coefficient of $\theta^{2}$, and equating to zero gives

$$
\left\langle q_{1234}^{2} q_{15}^{2}\right\rangle=\frac{3}{8}\left\langle q_{1234}^{2} q_{12}^{2}\right\rangle+\frac{5}{8}\left\langle q_{1234}^{2} q_{56}^{2}\right\rangle
$$


At the order four in $\beta^{\prime}$ we have leftovers from the order two, which thus vanish, and the coefficient of $\theta^{4}$ :

$$
\left\langle q_{1234}^{4}\right\rangle=\left\langle 16 q_{1234}^{2} q_{1235}^{2}-60 q_{1234}^{2} q_{1256}^{2}+80 q_{1234}^{2} q_{1567}^{2}-35 q_{1234}^{2} q_{5678}^{2}\right\rangle .
$$

In a similar way we obtain all the other relations, from higher orders, clearly equating to zero all the coefficients of the expansion in powers of $\theta$, with no need to expand in $\beta^{\prime}$.

When $\Phi=q_{1 \cdots s}^{2}$, notice that the relation we obtain from the lowest order in (17) is formally identical to equation (18), with zero in the left hand side, without the limit $\alpha^{\prime} \rightarrow \infty$. Hence, using the invariance with respect to permutations of replicas, we have the general form of the constraint which (14) and (15) are two special cases of. In general, for a suitable function $\phi_{1 \ldots s}$ of $s$ replicas, from Proposition 2 we can state

Theorem 5 Given an even integer s, the AC relation

$$
\left\langle\phi_{1 \ldots s} q_{1, s+1}^{2}\right\rangle=\frac{s-1}{2 s}\left\langle\phi_{1 \ldots s} q_{1,2}^{2}\right\rangle+\frac{s+1}{2 s}\left\langle\phi_{1 \ldots s} q_{s+1, s+2}^{2}\right\rangle
$$

holds.

Subtracting the equation above from the well known Ghirlanda-Guerra identity 12

$$
\left\langle\phi_{1 \ldots s} q_{1, s+1}^{2}\right\rangle=\frac{1}{s}\left\langle\phi_{1 \ldots s}\right\rangle\left\langle q_{1,2}^{2}\right\rangle+\frac{s-1}{s}\left\langle\phi_{1 \ldots s} q_{s+1, s+2}^{2}\right\rangle
$$

we get the other well known relation

$$
\left\langle\phi_{1 \ldots s} q_{s+1, s+2}^{2}\right\rangle=\frac{2}{s+1}\left\langle\phi_{1 \ldots s}\right\rangle\left\langle q_{12}^{2}\right\rangle+\frac{s-1}{s+1}\left\langle\phi_{1 \ldots s} q_{12}^{2}\right\rangle .
$$

While the Ghirlanda-Guerra identities are a consequence of the self-averaging, in the thermodynamic limit, of the energy density $H_{N} / N \equiv K$

$$
\left\langle K \phi_{s}\right\rangle=\langle K\rangle\left\langle\phi_{s}\right\rangle
$$

the AC relations are a consequence of stochastic stability, but they can be deduced from a self-averaging relation too:

$$
\mathbb{E} \Omega\left(K \phi_{s}\right)=\mathbb{E} \Omega(K) \Omega\left(\phi_{s}\right) .
$$


Clearly the third relation is hence a consequence of

$$
\mathbb{E} \Omega(K) \Omega\left(\phi_{s}\right)=\langle K\rangle\left\langle\phi_{s}\right\rangle .
$$

We stress that not only the energy density can be used to get the various relations, but several other quantities (as long as self-averaging is preserved) would do as well.

If we consider 4-overlaps, Proposition 2 gives

$$
\begin{array}{r}
\frac{(s-1)(s-2)(s-3)}{4 !}\left\langle\phi_{1 \ldots s} q_{1,2,3,4}^{2}\right\rangle-\frac{s(s-1)(s-2)}{3 !}\left\langle\phi_{1 \ldots s} q_{1,2,3, s+1}^{2}\right\rangle+ \\
\frac{(s-1) s(s+1)}{4}\left\langle\phi_{1 \ldots s} q_{1,2, s+1, s+2}^{2}\right\rangle-\frac{s(s+1)(s+2)}{3 !}\left\langle\phi_{1 \ldots s} q_{1, s+1, s+2, s+3}^{2}\right\rangle \\
+\frac{(s+1)(s+2)(s+3)}{4 !}\left\langle\phi_{1 \ldots s} q_{s+1, s+2, s+3, s+4}^{2}\right\rangle=0
\end{array}
$$

which again can be deduced from a self-averaging relation too [10, 9] and should be compared with the generalization of Ghirlanda-Guerra relations

$$
\begin{aligned}
& \frac{s(s-1)(s-2)(s-3)}{3 !}\left\langle q_{1,2,3,4} \phi\right\rangle- \frac{s(s-1)(s-2)}{2}\left\langle q_{1,2,3, s+1} \phi\right\rangle \\
&+\frac{(s-1) s(s+1)}{2 !}\left\langle q_{1,2, s+1, s+2} \phi\right\rangle-\frac{s(s+1)(s+2)}{3 !} \\
&\left\langle q_{1, s+1, s+2, s+3} \phi\right\rangle+\left\langle q_{1234}\right\rangle\langle\phi\rangle=0,
\end{aligned}
$$

which has been found in [9], as a consequence of the self-averaging of the energy density.

We do not write explicitly the general form of the constraints deduced from Proposition 2, as it is a very simple but tedious computation, which shows that the relations are in agreement with [10.

\subsection{Stochastic stability vs self-averaging}

Notice that there is a link between self-averaging quantities and quantities with zero derivative in (17), as they both lead to AC relations. One can see that self-averaging properties and stochastic stability are closely related, also by considering that self-averaging of proper quantities is typically formulated in terms of a suitable perturbation. In fact, let us consider 
two independent perturbations instead of just one in Lemma 3 We will use the same notation as in (13), and we will denote by $\Omega$ different measures, involving different perturbations, all vanishing in the thermodynamic limit, which is assumed to be interchangeable with the derivatives that we will take. Then the fundamental theorem of calculus can be used twice, to prove that

$$
\mathbb{E} \ln \Omega\left[\exp \left(-\beta \hat{H}\left(\alpha_{1}^{\prime}\right)-\beta \hat{H}\left(\alpha_{2}^{\prime}\right)\right)\right]=\left(\alpha_{1}^{\prime}+\alpha_{2}^{\prime}\right) A,
$$

where $A$ is of the same form as in (7), and from this relations one gets of course again the AC identities, like in Section 5 . From the equation above we immediately get

$$
\frac{\partial^{2}}{\partial \alpha_{1}^{\prime} \partial \alpha_{2}^{\prime}} \mathbb{E} \ln \Omega\left[\exp \left(-\beta_{1}^{\prime} \hat{H}\left(\alpha_{1}^{\prime}\right)-\beta_{2}^{\prime} \hat{H}\left(\alpha_{2}^{\prime}\right)\right)\right]=0
$$

but

$$
\begin{aligned}
\frac{\partial^{2}}{\partial \alpha_{1}^{\prime} \partial \alpha_{2}^{\prime}} \mathbb{E} \ln \Omega\left[\exp \left(-\beta \hat{H}\left(\alpha_{1}^{\prime}\right)-\beta \hat{H}\left(\alpha_{2}^{\prime}\right)\right)\right]=0 \\
=\mathbb{E} \ln \Omega\left[\exp \left(\beta_{1}^{\prime} J_{1}^{\prime} \sigma_{i_{1}} \sigma_{j_{1}}+\beta_{2}^{\prime} J_{2}^{\prime} \sigma_{i_{2}} \sigma_{j_{2}}\right]\right.
\end{aligned}
$$

and hence, a fortiori

$$
\begin{aligned}
\frac{\partial^{2}}{\partial\left(\beta_{1}^{\prime} J_{1}\right) \partial\left(\beta_{2}^{\prime} J_{2}\right)} \mathbb{E} \ln \Omega\left[\exp \left(\beta_{1}^{\prime} J_{1}^{\prime} \sigma_{i_{1}} \sigma_{j_{1}}+\beta_{2}^{\prime} J_{2}^{\prime} \sigma_{i_{2}} \sigma_{j_{2}}\right]=\right. \\
\mathbb{E} \Omega\left(\sigma_{i_{1}} \sigma_{j_{1}}\right)-\mathbb{E} \Omega\left(\sigma_{i_{1}}\right) \Omega\left(\sigma_{j_{1}}\right)=0 .
\end{aligned}
$$

This is a simple way to prove this kind of self-averaging. So on the one hand stochastic stability implies self-averaging and seems thus more general, on the other hand self-averaging can be formulated in three different types of factorizations, leading to all the three families of identities, and seems more versatile. But reasonings similar to that just illustrated can be put forward for the other two cases too. Moreover, Ghirlanda-Guerra identities can be shown to be a consequence of Quasi-Stationarity of Random Overlap Structures for Gaussian models, and the same can be extended to diluted spin glasses (work in progress with L.P. Arguin). 
As long as self-averaging only is considered, we refer to [9] for a systematic treatment.

\subsection{Revisiting the positivity of multi-overlaps}

We here hint at how to gain a better control of the phase transition discussed in section 4, using the expansion of the cavity streaming equation, to justify from a different perspective what is proven in [13: the fluctuations of the multi-overlaps diverge at lower temperatures as the number of replicas increases. This is a first step in the calculation of the critical exponents of the critical behavior of the multi-overlaps. We only sketch the reasonings, that proceed along the same lines described in [1].

We are going to prove that the first contribution to the average of the 2overlap in its $\tanh \left(\beta^{\prime}\right)=\theta$ expansion is of order two, while it is of order four for the 4-overlap, and so on for higher order multi-overlaps, as intuitively expected.

Let us write the streaming equation for $\left\langle q_{12}\right\rangle_{t}$, with $\beta^{\prime}=\beta, \alpha^{\prime}=\alpha$

$$
\partial_{t}\left\langle q_{12}\right\rangle_{t}=\alpha \theta^{2}\left\langle q_{12}^{2}-4 q_{12} q_{23}+3 q_{12} q_{34}\right\rangle_{t}+O\left(\theta^{4}\right) .
$$

But $\left\langle q_{12}^{2}\right\rangle_{t}=\left\langle q_{12}^{2}\right\rangle$ because $q_{12}^{2}$ is a filled monomial, and it can be integrated offering

$$
\left\langle q_{12}\right\rangle_{t}=\alpha \theta^{2}\left\langle q_{12}^{2}\right\rangle t+\alpha \theta^{2} \int_{0}^{1} d t\left(\left\langle q_{12} q_{23}\right\rangle_{t}+3\left\langle q_{12} q_{34}\right\rangle_{t}\right)+O\left(\theta^{4}\right) .
$$

We now prove that the terms under the integral are of higher order in $\theta$. It is enough to notice that such terms are fillable but not filled, so we can expand them using the streaming equation to evaluate their order of contribution, which starts from $\theta^{3}$ as can be deduced from the expansions reported in Appendix B. The same approach applies to the 4-overlap, in fact we can write

$$
\begin{gathered}
\partial_{t}\left\langle q_{1234}\right\rangle_{t}=\alpha \theta^{2}\left\langle 10 q_{1234} q_{56}-16 q_{1234} q_{15}+6 q_{1234} q_{12}\right\rangle_{t}+ \\
\alpha \theta^{4}\left\langle q_{1234}^{2}-16 q_{1234} q_{1235}+60 q_{1234} q_{1256}-80 q_{1234} q_{1567}-35 q_{1234} q_{5678}\right\rangle_{t}+O\left(\theta^{6}\right)
\end{gathered}
$$


It can be readily noticed that the only contribution at the fourth order is due to $\left\langle q_{1234}^{2}\right\rangle$, being the only filled monomial. The calculations in Appendix $B$ show that the three contributions from the second order in $\theta$ (i.e. $\left.q_{1234} q_{56}, q_{1234} q_{15}, q_{1234} q_{12}\right)$ contribute at orders higher than four, so the first term in the four-replica multi-overlap expansion is positive (being a square), consistently with what we showed in section 4 . We notice that, while the first contribution to the 2-overlap is of order two in $\theta$, the first contribution to the 4-overlap is of order four, and analogously for the higher order multi-overlaps. So it is not surprising that at the point where the 2-overlap fluctuations start diverging, the fluctuations of the 4-overlap do not, and so on for higher orders, consistently with what is proven in [13.

In Appendix B are reported all the calculations needed for the lowest orders.

\section{Conclusion and outlook}

We proved the validity of Aizenman-Contucci relations for dilute spin glasses, and we exhibited further relations for multi-overlaps. Some more general relations can be found with the same stochastic stability methods for internal energy, but also from the cavity part of the RaMOSt trial function, by means of a control of the response of the average of generic observables with respect to the change of a perturbing parameter. We also showed that the multi-overlaps undergo the same transition the 2-overlap exhibits in fully connected models, i.e. it remains strictly positive, below the critical temperature, if we apply an external field and then remove it in the thermodynamic limit. The external field is properly modulated, in diluted systems, by the degree of connectivity of the perturbation.

The further natural development is the study of the extension to multioverlaps of the self-averaging identities (known as Ghirlanda-Guerra in fully connected models) to prove that even multi-overlaps are non-negative with probability one in dilute spin glasses (the identities will appear in [9]). This 
would extend to odd-spin interactions the replica bounds so far rigorously valid only for even interactions, and such a result would be important for the application of dilute spin glasses to optimization problems like the KSAT.

Another development of the current work is the calculation of the critical exponents of the multi-overlaps, which has been gained for fully connected models in [1] with the same techniques here shown to be fruitful in dilute models too.

\section{A Formulas for the RaMOSt expansions}

Let us report for convenience the well known expansion

$$
\tanh (x) \sum \frac{2^{2 n}}{2 n !}\left(2^{2 n}-1\right) B_{2 n} x^{2 n-1}
$$

where $B_{n}$ and the Bernoulli numbers, defined by

$$
\frac{x}{e^{x}-1}=\sum \frac{B_{n} x^{n}}{n !}
$$

so that

$$
\tanh (\beta)=\beta-\frac{1}{3} \beta^{3}+\frac{2}{15} \beta^{5}-\frac{17}{315} \beta^{7}+\frac{62}{2835} \beta^{9} \cdots .
$$

We also report here the result the computation below, that we used in the expansions of the previous sections.

Order two:

$$
\begin{aligned}
& \mathbb{E} \Omega\left(\hat{H}^{2}\right)=\alpha^{\prime} \\
& \mathbb{E} \Omega^{2}(\hat{H})=\alpha^{\prime}\left\langle q_{12}^{2}\right\rangle
\end{aligned}
$$


Order four:

$$
\begin{aligned}
& \mathbb{E} \Omega\left(\hat{H}^{4}\right)=\alpha^{\prime}+\alpha^{\prime 2} 3 \\
& \mathbb{E} \Omega\left(\hat{H}^{3}\right) \Omega(\hat{H})=\alpha^{\prime}\left\langle q_{12}^{2}\right\rangle+\alpha^{\prime 2} 3\left\langle q_{12}^{2}\right\rangle \\
& \mathbb{E} \Omega^{2}\left(\hat{H}^{2}\right)=\alpha^{\prime}+\alpha^{\prime 2}\left(1+2\left\langle q_{12}^{4}\right\rangle\right) \\
& \mathbb{E} \Omega\left(\hat{H}^{2}\right) \Omega^{2}(\hat{H})=\alpha^{\prime}\left\langle q_{12}^{2}\right\rangle+\alpha^{\prime 2}\left(\left\langle q_{12}^{2}\right\rangle+2\left\langle q_{12}^{2} q_{13}^{2}\right\rangle\right) \\
& \mathbb{E} \Omega^{4}(\hat{H})=\alpha^{\prime}\left\langle q_{1234}^{2}\right\rangle+\alpha^{\prime 2} 3\left\langle q_{12}^{2} q_{34}^{2}\right\rangle
\end{aligned}
$$

Order six:

$$
\begin{aligned}
& \mathbb{E} \Omega\left(\hat{H}^{6}\right)=\alpha^{\prime}+\alpha^{\prime 2} 15+\alpha^{\prime 3} 15 \\
& \mathbb{E} \Omega\left(\hat{H}^{5}\right) \Omega(\hat{H})=\alpha^{\prime}\left\langle q_{12}^{2}\right\rangle+\alpha^{\prime 2} 15\left\langle q_{12}^{2}\right\rangle+\alpha^{\prime 3} 15\left\langle q_{12}^{2}\right\rangle \\
& \mathbb{E} \Omega\left(\hat{H}^{4}\right) \Omega\left(\hat{H}^{2}\right)=\alpha^{\prime}+\alpha^{\prime 2}\left(7+8\left\langle q_{12}^{4}\right\rangle\right)+\alpha^{\prime 3}\left(3+12\left\langle q_{12}^{4}\right\rangle\right) \\
& \mathbb{E} \Omega^{2}\left(\hat{H}^{3}\right)=\alpha^{\prime}\left\langle q_{12}^{2}\right\rangle+\alpha^{\prime 2} 15\left\langle q_{12}^{2}\right\rangle+\alpha^{\prime 3}\left(9\left\langle q_{12}^{2}\right\rangle+6\left\langle q_{12}^{6}\right\rangle\right) \\
& \mathbb{E} \Omega(\hat{H}) \Omega\left(\hat{H}^{2}\right) \Omega\left(\hat{H}^{3}\right)=\alpha^{\prime}\left\langle q_{12}^{2}\right\rangle+\alpha^{\prime 2}\left(7\left\langle q_{12}^{2}\right\rangle+8\left\langle q_{12}^{2} q_{23}^{2}\right\rangle\right) \\
& \quad+\alpha^{\prime 3}\left(3\left\langle q_{12}^{2}\right\rangle+6\left\langle q_{12}^{2} q_{13}^{2}\right\rangle+6\left\langle q_{12}^{2} q_{13}^{4}\right\rangle\right)
\end{aligned}
$$




\section{B Formulas for the cavity expansions}

$$
\begin{aligned}
& \partial_{t}\left\langle q_{12}\right\rangle_{t}=\alpha \theta^{2}\left\langle q_{12}^{2}-4 q_{12} q_{23}+3 q_{12} q_{34}\right\rangle_{t}+O\left(\theta^{4}\right) \\
& \partial_{t}\left\langle q_{12} q_{23}\right\rangle_{t}=\alpha \theta^{2}\left\langle 6 q_{12} q_{23} q_{45}-6 q_{12} q_{23} q_{14}-3 q_{12} q_{23} q_{24}+q_{12} q_{23} q_{13}\right. \\
& \left.+2 q_{12}^{2} q_{23}\right\rangle+\alpha \theta^{4}\left\langle 15 q_{12} q_{23} q_{4567}-20 q_{12} q_{23} q_{1456}-10 q_{12} q_{23} q_{2456}\right. \\
& \left.+12 q_{12} q_{23} q_{1245}+6 q_{12} q_{23} q_{1345}-3 q_{12} q_{23} q_{1234}\right\rangle_{t}+O\left(\theta^{6}\right) \\
& \partial_{t}\left\langle q_{12} q_{34}\right\rangle_{t}=\alpha \theta^{2}\left\langle 10 q_{12} q_{34} q_{56}-16 q_{12} q_{34} q_{15}+2 q_{12}^{2} q_{34}+4 q_{12} q_{34} q_{13}\right\rangle_{t} \\
& +\alpha \theta^{4}\left\langle 35 q_{12} q_{34} q_{5678}-80 q_{12} q_{34} q_{1567}+20 q_{12} q_{34} q_{1256}+40 q_{12} q_{34} q_{1356}\right. \\
& \left.-16 q_{12} q_{34} q_{1235}+q_{12} q_{34} q_{1234}\right\rangle_{t}+O\left(\theta^{6}\right) \\
& \partial_{t}\left\langle q_{1234}\right\rangle_{t}=\alpha \theta^{2}\left\langle 10 q_{1234} q_{56}-16 q_{1234} q_{15}+6 q_{1234} q_{12}\right\rangle_{t}+\alpha \theta^{4}\left\langle q_{1234}^{2}\right. \\
& \left.-16 q_{1234} q_{1235}+60 q_{1234} q_{1256}-80 q_{1234} q_{1567}-35 q_{1234} q_{5678}\right\rangle_{t}+O\left(\theta^{6}\right) \\
& \partial_{t}\left\langle q_{1234} q_{12}\right\rangle_{t}=\alpha \theta^{2}\left\langle 10 q_{1234} q_{12} q_{56}-8 q_{1234} q_{12} q_{15}\right. \\
& \left.-8 q_{1234} q_{12} q_{35}+q_{1234} q_{12}^{2}+5 q_{1234} q_{12} q_{13}\right\rangle_{t}+\alpha \theta^{4}\left\langle 35 q_{1234} q_{12} q_{5678}\right. \\
& -40 q_{1234} q_{12} q_{1567}-40 q_{1234} q_{12} q_{3567}+10 q_{1234} q_{12} q_{1256}+40 q_{1234} q_{12} q_{1356} \\
& \left.+10 q_{1234} q_{12} q_{3456}-16 q_{1234} q_{12} q_{1235}+q_{1234}^{2} q_{12}\right\rangle_{t}+O\left(\theta^{6}\right) \\
& \partial_{t}\left\langle q_{1234} q_{56}\right\rangle_{t}=\alpha \theta^{2}\left\langle 15 q_{1234} q_{15} q_{67}-15 q_{1234} q_{15} q_{26}-10 q_{1234} q_{15} q_{56}\right. \\
& \left.+5 q_{1234} q_{15} q_{12}+4 q_{1234} q_{15} q_{23}+q_{1234} q_{15}^{2}\right\rangle_{t}+\alpha \theta^{4}\left\langle 70 q_{1234} q_{15} q_{6789}\right. \\
& -105 q_{1234} q_{15} q_{2678}-70 q_{1234} q_{15} q_{1678}+15 q_{1234} q_{15} q_{1567}+90 q_{1234} q_{15} q_{1267} \\
& +45 q_{1234} q_{15} q_{2367}+20 q_{1234} q_{15} q_{1235}-25 q_{1234} q_{15} q_{1246} \\
& \left.-5 q_{1234} q_{15} q_{2346}+4 q_{1234} q_{15} q_{1236}+q_{1234}^{2} q_{15}\right\rangle_{t}+O\left(\theta^{6}\right) \\
& \partial_{t}\left\langle q_{1234} q_{56}\right\rangle_{t}=\alpha \theta^{2}\left\langle 21 q_{1234} q_{56} q_{78}-24 q_{1234} q_{56} q_{17}\right. \\
& \left.-12 q_{1234} q_{56} q_{57}+6 q_{1234} q_{56} q_{12}+8 q_{1234} q_{56} q_{57}+q_{1234} q_{56}^{2}\right\rangle \\
& +\alpha \theta^{4}\left\langle 126 q_{1234} q_{56} q_{7890}-112 q_{1234} q_{56} q_{5789}-224 q_{1234} q_{56} q_{1789}\right. \\
& +21 q_{1234} q_{56} q_{5678}+168 q_{1234} q_{56} q_{1578}+126 q_{1234} q_{56} q_{1378}-24 q_{1234} q_{56} q_{3567} \\
& \left.+14 q_{1234} q_{56} q_{1235}+q_{1234}^{2} q_{56}\right\rangle_{t}+O\left(\theta^{6}\right)
\end{aligned}
$$




\section{References}

[1] A. Agostini, A. Barra, L. De Sanctis, Positive-Overlap Transition and Critical Exponents in Mean Field Spin Glasses, to appear in J. Stat. Mech.. ArXiv:cond-mat/0609254.

[2] M. Aizenman, P. Contucci, On the stability of the quenched state in mean field spin glass models, J. Stat. Phys. 92, 765 (1998).

[3] M. Aizenman, A. Ruzmaikina, Characterization of invariant measures at the leading edge for competing particle systems, Ann. Probab. 33, no. 1, 82-113, 2005.

[4] M. Aizenman, R. Sims, S. L. Starr, Mean-Field Spin Glass models from the Cavity-ROSt Perspective. ArXiv: math-ph/0607060.

[5] A. Barra, Irreducible free energy expansion for mean field spin glass model, J. Stat. Phys. (2006). DOI: 10.1007/s10955-005-9006-6.

[6] A. Barra, L. De Sanctis, Overlap Fluctuations from Random Overlap Structures, J. Math. Phys. 47-10, at press. ArXiv:cond-mat/0607615.

[7] L. De Sanctis, Random Multi-Overlap Structures and Cavity Fields in Diluted Spin Glasses, J. Stat. Phys. 117 785-799 (2004).

[8] L. De Sanctis, Ultrametric Broken Replica Symmetry RaMOSt, J. Stat. Phys. 122-5 857-874 (2006).

[9] L. De Sanctis, S. Franz, Self-averaging identities for dilute spin glasses, to be submitted.

[10] S. Franz, M. Leone, F.L. Toninelli, Replica bounds for diluted nonPoissonian spin systems, J. Phys. A 3610967 (2003).

[11] S. Franz, M. Mezard, G. Parisi, L. Peliti, The response of glassy systems to random perturbations: a bridge between equilibrium and offequilibrium, J. Stat. Phys. 97459 (1999). 
[12] S. Ghirlanda, F. Guerra, General properties of overlap distributions in disordered spin systems. Towards Parisi ultrametricity, J. Phys. A, 31 9149-9155 (1998).

[13] F. Guerra, F.L. Toninelli, The high temperature region of the VianaBray diluted spin glass model, J. Stat. Phys. 115 (2004).

[14] G. Parisi, F. Ricci-Tersenghi, On the origin of ultrametricity, J. Phys. A: Math. Gen. 33113 (2000)

[15] M. Talagrand, Spin glasses: a challenge for mathematicians. Cavity and Mean field models, Springer Verlag (2003). 